\title{
Prehospital triage of patients diagnosed with perforated peptic ulcer or peptic ulcer bleeding: an observational study of patients calling 1-1-2
}

Kasper Bonnesen ${ }^{1 *}$ D, Kristian D. Friesgaard ${ }^{1}$, Morten T. Boetker ${ }^{1,2}$ and Lone Nikolajsen ${ }^{2}$

\begin{abstract}
Background: Triage systems are used in emergency medical services to systematically prioritize prehospital resources according to individual patient conditions. Previous studies have shown cases of preventable deaths in emergency medical services even when triage systems are used, indicating a potential undertriage among some conditions. The aim of this study was to investigate the triage level among patients diagnosed with perforated peptic ulcer (PPU) or peptic ulcer bleeding (PUB).

Methods: In a three-year period in Central Denmark Region, all patients hospitalized within $24 \mathrm{~h}$ after a 1-1-2 emergency call and who subsequently received either a PPU or a PUB (hereinafter combined and referred to as PPU/PUB) or a First Hour Quintet (FHQ: respiratory failure, stroke, trauma, cardiac chest pain, and cardiac arrest) diagnosis were investigated. A modified Poisson regression was used to estimate the relative risk of receiving the highest and lowest prehospital response level. Also, a linear regression analysis was used to estimate the relative risk of 30-day mortality.

Results: Of 8658 evaluated patients, 263 were diagnosed with PPU/PUB. After adjusting for relevant confounding variables, patients diagnosed with PPU/PUB were less likely to receive ambulance transportation compared to patients diagnosed with stroke, $\mathrm{RR}=1.41(\mathrm{Cl}$ : 1.28-1.56); trauma, $\mathrm{RR}=1.28$ (Cl: 1.15-1.42); cardiac chest pain, $\mathrm{RR}=1.47$ (Cl: 1.33-1.62); and cardiac arrest, $\mathrm{RR}=1.44$ (Cl: 1.31-1.42). Among patients diagnosed with PPU/PUB, 6.5\% (Cl: 3.3-9.7) did not receive ambulance transportation. The proportion of patients not receiving ambulance transportation was higher among patients diagnosed with PPU/PUB compared to patients diagnosed with an FHQ diagnosis. The 30-day mortality rate among patients diagnosed with PPU/PUB was 7.8\% (Cl: 4.2-11.1). This was lower than the 30-day mortality rate among patients diagnosed with respiratory failure $(P=0.010)$, stroke $(P=0.001)$, and cardiac arrest $(P<0.001)$, but comparable to the 30-day mortality among patients diagnosed with cardiac chest pain $(P=0.080)$ and trauma $(P=0.281)$.

Conclusion: Among patients calling 1-1-2, fewer patients diagnosed with PPU/PUB received ambulance transportation than patients diagnosed with FHQ diagnoses, despite a high mortality among patients diagnosed with PPU/PUB.
\end{abstract}

Keywords: Perforated peptic ulcer, Peptic ulcer bleeding, Prehospital, Triage

\footnotetext{
* Correspondence: kasbon@rm.dk

${ }^{1}$ Research Department, Prehospital Emergency Medical Services, Olof Palmes

Allé 34, 8200 Aarhus N, Central Denmark Region, Denmark

Full list of author information is available at the end of the article
} 


\section{Background}

Triage systems are used in emergency medical services (EMS) for systematic prioritization of prehospital resources according to the presumed severity and urgency of the individual patient's condition. Rapid diagnostics and treatment play a key role in severe time-critical conditions. Thus, avoiding undertriage is important. The criteria-based Danish Index for emergency care is used after all emergency calls (1-1-2) to an emergency medical communication center (EMCC) in Denmark. The Danish Index generally triages patients with the highest hospital admission risks and case fatality rates to the highest level of emergency [1]. A previous Danish study suggested cases of preventable deaths in EMS despite applying the Danish Index, thus indicating a potential undertriage among some conditions [2]. This has also been demonstrated in a Finnish EMS system [3]. The First Hour Quintet (FHQ) diagnoses (respiratory failure, stroke, trauma, cardiac chest pain, and cardiac arrest), are highly targeted in prehospital triage systems [4]. However, identifying other high-risk diseases may discover conditions susceptible for undertriage.

Diseases of the digestive system account for about $3 \%$ of diagnoses among patients hospitalized after a 1-1-2 call. However, they rarely receive the highest-level response (i.e. ambulance with lights and sirens) [1,5]. Patients with the severe condition perforated peptic ulcer (PPU) have a 30day mortality rate of more than $20 \%$, and for patients with peptic ulcer bleeding (PUB) the rate is nearly $10 \%[6,7]$. To reduce mortality, national inhospital guidelines implemented nationwide recommend reduced time from hospital admission to diagnosis and treatment for these patients $[8,9]$. However, versatile symptomatology (e.g. referred abdominal pain, anemia-caused tiredness, etc.) might lead to inadequate symptom description [10], making it difficult to raise a correct suspicion of PPU and PUB (hereinafter combined and referred to as PPU/PUB) after the medical emergency call. A combination of high short-term mortality and low prehospital triage level of patients with PPU/PUB may suggest a need for improvements in emergency care for these patients.

Therefore, the aim of this study was to examine prehospital triage among patients diagnosed inhospitally with PPU/PUB and compare these findings with the triage among other severe time-critical conditions. We hypothesize that patients diagnosed with PPU/PUB are less likely to receive the most acute level of triage despite mortality rates comparable to FHQ patients.

\section{Methods}

\section{Study population and setting}

This was a population-based observational study performed in the Central Denmark Region in a threeyear period from December 1, 2011 to November 30, 2014. The Central Denmark Region covers an area of $13,007 \mathrm{~km}^{2}$ urban, suburban, and rural land with 1.3 million inhabitants corresponding to $23 \%$ of the Danish population [11].

We included all first time 1-1-2 calls within the study period. Exclusion criteria included: invalid civil personal register $(\mathrm{CPR})$ number, if patients called more than one time in the study period, and non-existing symptom categories within the dispatch protocol. The cohort was identified via technical dispatch software in the EMCC, containing data on 1-1-2 calls, triage level, and prehospital time stamps. Vital status, gender, and age were retrieved from the Danish Civil Registration System [12], and data on previous diseases and present diagnoses, according to the 10th version of the International Classification of Disease (ICD-10), were retrieved from the National Patient Register [13]. Each Danish citizen has a unique CPR number that makes it possible to link Danish registers on an individual level. Patients were followed from hospital admission date to either death, emigration, or November 30, 2014 - whichever came first. The study was approved by the Danish Data Protection Agency (record number 1-16-02-207-15). Approval by the local ethics committee and collection of informed consent are not required for observational studies.

\section{Triage}

The Danish health care system provides free and unconstrained access for all citizens to general practitioners, prehospital emergency medical services, and hospitals [14]. Thus, patients diagnosed with PPU/PUB or a FHQ diagnosis can gain access to acute medical help either via general practitioners, through the Danish national emergency number 1-1-2, or by appearance at a hospital (which is rare). When people dial 1-1-2, they are connected to a healthcare professional in the EMCC. In the Central Denmark Region, the EMCC is staffed by registered nurses and paramedics with six weeks' additional training in communication and use of the dispatch protocol Danish Index [15]. This tool is designed to evaluate the severity and urgency of the patients' conditions. It is divided into 37 symptom chapters (e.g. non-traumatic bleeding, stomach or back pain, traffic accident, etc.), each one subdivided into 5 levels of decreasing emergency (A-E).

PPU/PUB was defined according to specific ICD-10 diagnosis-codes listed by the Danish Clinical Register of Emergency Surgery [16]. Respiratory failure, stroke, cardiac chest pain, and cardiac arrest were defined according to specific ICD-10 diagnosis-codes listed by the European Emergency Data Project [17]. Trauma was defined as ICD-10 trauma diagnoses with an inhospital survival probability $\leq 0.941$ based on pooled data from nearly 4 million injuries in seven industrialized countries, including Denmark, with similar emergency care setups [18, 19]. No validation of the trauma diagnoses has been conducted 
whereas all other diagnoses have been validated previously $[20,21]$. The exact definition of each group is listed in the Appendix 3 (Table 5).

\section{Statistical analyses}

All statistical analyses were conducted in STATA version 14.1 (StataCorp, TX, USA). Categorical data were presented as number and percentage (\%) with a $95 \%$ confidence interval $(\mathrm{CI})$. Comparisons of categorical data were made by a chi-squared test. Continuous data were presented as means with a 95\% CI for normally distributed data and as medians with interquartile ranges (IQR) for skewed data. Comparisons of continuous data were made using a student's $t$ test or a Mann-Whitney U test when appropriate.

A modified Poisson regression with a robust errorvariance approach was used to estimate the relative risks (RR) of level A triage and level E triage [22]. PPU/PUB was used as a reference point and the following covariates were included in the adjusted analyses: age, sex, Charlson Comorbidity Index (CCI) score, and time of 1-1-2 call.

30-day mortality was used as proxy of disease severity. The initially intended use of a Cox proportional regression analysis was abandoned, as the data did not fulfill the proportional hazards assumption. Instead, a generalized linear regression of pseudo-observations was conducted to achieve relative risk estimates of mortality at specific time points. This kind of statistics does not require the fulfillment of the proportional hazards assumption and was therefore applicable [23]; the primary analysis was conducted on complete cases. In a sensitivity analysis, we repeated the regression analysis on triage level after imputing missing data by following two models: a multiple imputation model using chained equations and a bootstrapping model [24, 25].

\section{Results}

In the three-year study period, 136,891 1-1-2 emergency calls were received by the EMCC in the Central Denmark Region. Figure 1 displays a flowchart of the patient inclusion. Of the 94,881 patients who fulfilled the inclusion criteria, 8658 (6.32\%) were diagnosed with PPU/PUB or a FHQ diagnosis. Patients diagnosed with PPU/PUB were older and had more comorbidities than the average FHQ patient. The variation of potential confounding variables between PPU/PUB and FHQ diagnoses is displayed in Table 1. Among the 8658 patients investigated, 263 were diagnosed with PPU/PUB. Of these, $63.4 \%$ (CI: 57.1-69.6) received a level A response. After adjusting for age, sex, CCI score, and time of 1-1-2 call, the study showed that patients diagnosed with PPU/ PUB were less likely to receive a level A triage compared to patients diagnosed with stroke, $\mathrm{RR}=1.41$ (CI: 1.28-1.56); trauma, $\mathrm{RR}=1.28$ (CI: 1.15-1.42); cardiac chest pain, $\mathrm{RR}=$ 1.47 (CI: 1.33-1.62); and cardiac arrest, RR $=1.44$ (CI: 1.311.42). On the contrary, the risk of level $E$ triage was higher among patients diagnosed with PPU/PUB compared to patients diagnosed with a FHQ diagnosis except for patients diagnosed with respiratory failure, $(\mathrm{RR}=0.60$ (CI: $0.35-1.05$ ) , Table 2). The association between diagnosis-groups and triage was not noticeably modified by age, sex, and CCI score (Table 3 in Appendix 1). After imputing missing data on triage level, patients diagnosed with PPU/PUB had a lower risk of receiving a level A triage compared to all FHQ diagnoses including respiratory failure. After imputation the results regarding level E triage remained robust, except in the bootstrapping model, where patients diagnosed with stroke had a comparable possibility of receiving a level E triage (see Table 4 in the Appendix 2).

The 30-day mortality rate among patients diagnosed with PPU/PUB was 7.8\% (CI: 4.2-11.1). In the adjusted analysis, 30-day mortality was similar for patients with cardiac chest pain, $\mathrm{RR}=0.64$ (CI: 0.38-1.06) and patients diagnosed with trauma, $\mathrm{RR}=1.33$ (CI: $0.72-2.44$ ), but higher for respiratory failure, $\mathrm{RR}=1.67$ (CI: 1.06-2.64); stroke, $\mathrm{RR}=2.03$ (CI: 1 . 27-3.24), and cardiac arrest, $\mathrm{RR}=6.72$ (CI: 4.21-10.73).

\section{Discussion}

In this large observational study, including 8658 patients hospitalized within $24 \mathrm{~h}$ from a 1-1-2 call and diagnosed with PPU/PUB or a FHQ diagnosis, we found that the 30-day mortality rate among PPU/PUB patients was comparable to two of the five FHQ groups but fewer patients with PPU/ PUB received level A triage compared to the FHQ patients.

The main objective of the EMCC is to dispatch the correct level of triage to keep the degree of undertriage to a minimum, as undertriage is associated with increased mortality. Andersen et al. discovered 18 potentially preventable deaths same day as the 1-1-2 call in an 18-month period [2]. Kuisma et al. also discovered 29 potentially avoidable deaths and one definitely avoidable death in patients receiving the lower urgency triage categories in a three-year period [3] and other studies have shown similar results [26-29]. The possibility of reducing the number of potential cases of undertriage seems present according to previous studies. A Belgium study has shown that two training sessions can increase the sensitivity of sending a mobile critical care unit along with a basic life support ambulance from $36 \%$ to $60 \%$ without any change in specificity [30]. Another Belgium study suggested trends towards an increased ability to obtain information from emergency callers among the telephone responders after a training session [31]. An American study showed that appropriate performance feedback could increase dispatch protocol compliance from $76 \%$ to $95 \%$ [32].

Today, the telephone responders in the EMCC are offered continuous education on a regular basis. However, a more systematic approach might be beneficial. The high amount of level A triage among patients diagnosed with cardiac chest pain (primarily acute myocardial infarction and angina pectoris) seen in our study might be 


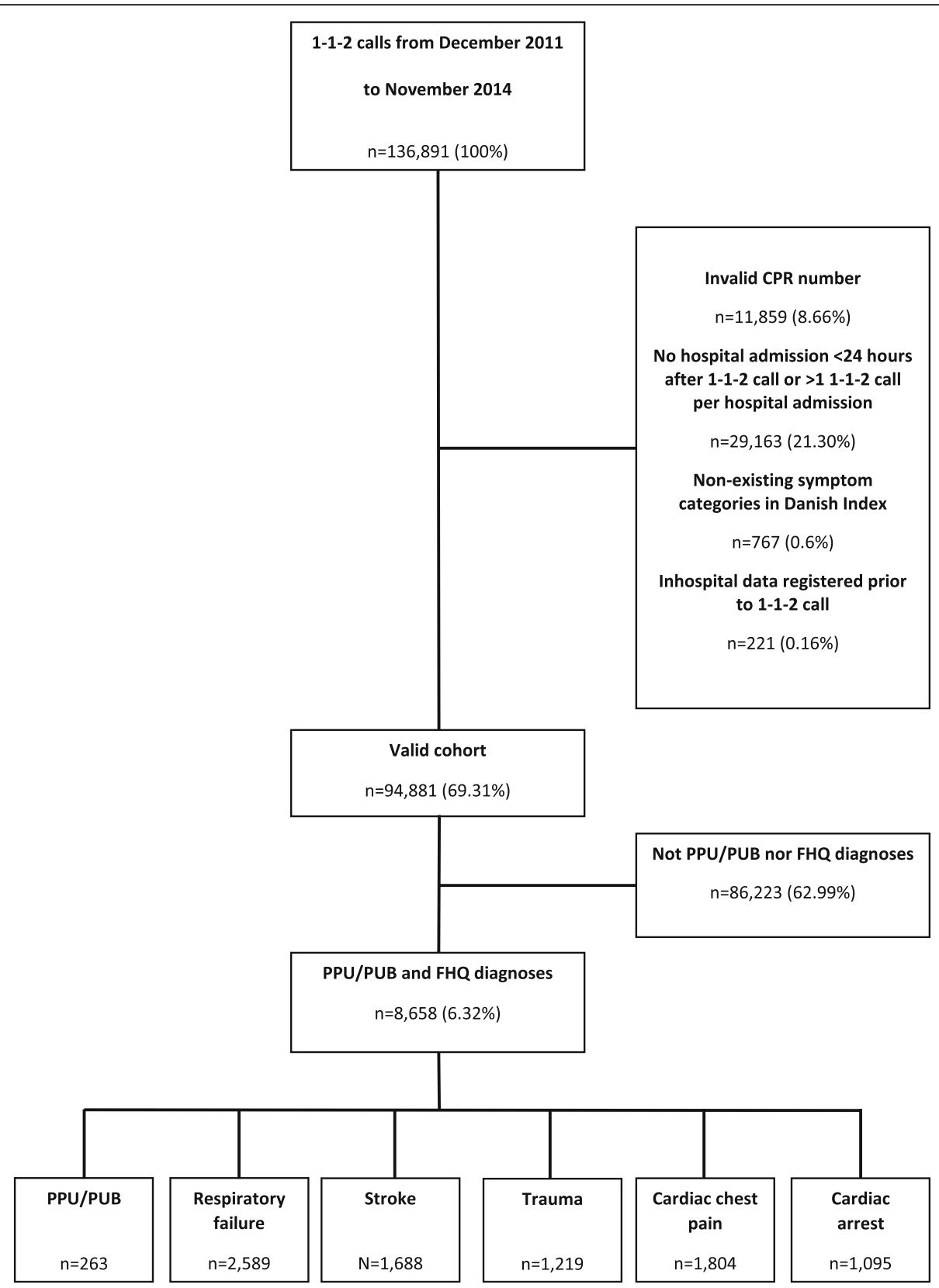

Fig. 1 Flowchart of patient inclusion

an effect of previous research regarding patients diagnosed with ST-elevation myocardial infarction, causing a change in procedure for these patients. Today, STelevation myocardial infarction is diagnosed prehospitally and the patients are field-triaged directly to an invasive center. This has resulted in reduced time consumption of reperfusion, a decrease in mortality, and a lower risk of congestive heart failure [33-36]. On the contrary, increased attention to patients presenting themselves with chest pain may potentially result in overtriage [37, 38].
The main strength of this study is its large-sized population-based cohort, which improves precision and external validity. The unique CPR numbers provide the possibility of record linkage of validated registers on an individual level $[12,39]$. Another strength is the free access to health care for all patients mitigating the risk of selection bias. Other studies might have an underrepresentation of less critical illnesses due to treatment expenses. Furthermore, diagnoses are validated for most ICD-10 diagnoses and this ensures correct classification. The nearly complete data set of the included covariates is also a strength. On the 


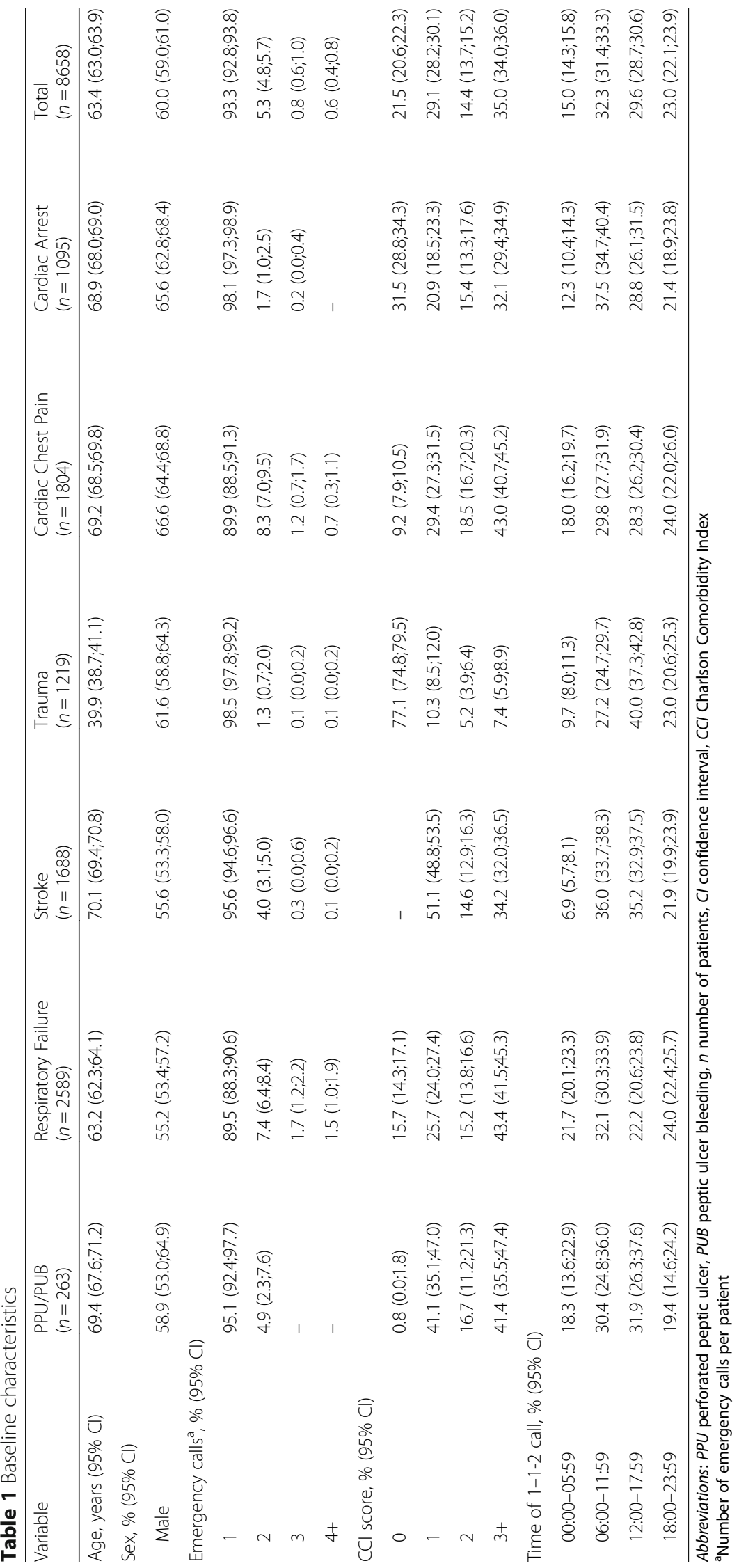


Table 2 Triage and mortality

\begin{tabular}{|c|c|c|c|c|c|}
\hline & Group & Frequency, \% (95\% Cl) & Unadjusted, RR (95\% Cl) & Adjusted $^{a, b}$, RR $(95 \%$ Cl) & $p$-value \\
\hline \multirow[t]{6}{*}{ A-triage $(n=7538)$} & PPU/PUB & $63.26(57.12-69.61)$ & 1 (ref.) & 1 (ref.) & - \\
\hline & Respiratory Failure & $69.73(67.85-71.62)$ & $1.10(0.99-1.22)$ & $1.09(0.98-1.20)$ & 0.105 \\
\hline & Stroke & 89.22 (87.67-90.78) & $1.41(1.27-1.56)$ & $1.41(1.28-1.56)$ & $<0.001$ \\
\hline & Trauma & $83.81(81.46-86.16)$ & $1.32(1.19-1.46)$ & $1.28(1.15-1.42)$ & $<0.001$ \\
\hline & Cardiac Chest Pain & 93.22 (91.99-94.45) & $1.47(1.33-1.62)$ & $1.46(1.33-1.62)$ & $<0.001$ \\
\hline & Cardiac Arrest & $92.23(90.52-93.95)$ & $1.46(1.32-1.61)$ & $1.44(1.30-1.59)$ & $<0.001$ \\
\hline \multirow[t]{6}{*}{ E-triage $(n=7538)$} & PPU/PUB & $6.47(3.28-9.65)$ & 1 (ref.) & 1 (ref.) & - \\
\hline & Respiratory Failure & $3.79(3.01-4.58)$ & $0.59(0.35-1.00)$ & $0.60(0.35-1.05)$ & 0.073 \\
\hline & Stroke & $2.56(1.77-3.36)$ & $0.40(0.22-0.71)$ & $0.41(0.23-0.73)$ & 0.002 \\
\hline & Trauma & $0.74(0.19-1.29)$ & $0.11(0.05-0.28)$ & $0.13(0.05-0.36)$ & $<0.001$ \\
\hline & Cardiac Chest pain & $1.62(1.00-2.24)$ & $0.25(0.13-0.47)$ & $0.25(0.13-0.47)$ & $<0.001$ \\
\hline & Cardiac Arrest & $1.17(0.50-1.86)$ & $0.18(0.08-0.39)$ & $0.18(0.08-0.40)$ & $<0.001$ \\
\hline \multirow[t]{6}{*}{ 30-day mortality $(n=7538)$} & PPU/PUB & $7.83(4.52-11.13)$ & 1 (ref.) & 1 (ref.) & - \\
\hline & Respiratory Failure & $12.16(10.87-13.44)$ & $1.55(1.01-2.39)$ & $1.67(1.06-2.64)$ & 0.028 \\
\hline & Stroke & $16.83(15.01-18.66)$ & $2.15(1.39-3.32)$ & $2.03(1.27-3.24)$ & 0.003 \\
\hline & Trauma & $3.16(2.16-4.17)$ & $0.40(0.24-0.68)$ & $1.33(0.72-2.44)$ & 0.364 \\
\hline & Cardiac Chest Pain & $4.45(3.49-5.42)$ & $0.57(0.35-0.91)$ & $0.64(0.38-1.06)$ & 0.080 \\
\hline & Cardiac Arrest & $47.34(40.67-54.00)$ & 6.05 (3.89-9.41) & $6.72(4.21-10.73)$ & $<0.001$ \\
\hline
\end{tabular}

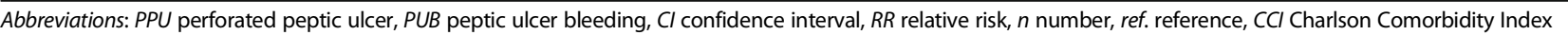
${ }^{\text {a }}$ Triage adjusted for age, sex, $\mathrm{CCl}$ score, and time of 1-1-2 call

$\mathrm{b}_{30}$-day mortality adjusted for age, sex, $\mathrm{CCl}$ score, and time of 1-1-2 call - an interaction term between exposure and sex was included

other hand, the weaknesses of this study relate to its register-based observational design. First of all, the proportion of patients with invalid CPR contributes to potential selection bias. However, missing data on emergency patients making a 1-1-2 call is difficult to avoid, as seen in other similar observational prehospital studies $[1,4$, 40-42]. Compared to the final cohort, patients with invalid CPR had a higher proportion of level $\mathrm{E}$ triage and tended to call more frequently in the evening and at night. Further information on these patients was unobtainable, thus it remains unanswered whether these patients differed from the final cohort in other ways. Second, missing outcome data on triage level, especially among patients diagnosed with trauma, may eventually lead to bias. In order to address this issue, a sensitivity analysis with imputed triage level was conducted using two different imputation models, and no major estimate changes were observed. Last, although adjusted for several potential confounders, unobserved confounding would still be able to affect our estimates, and residual confounding cannot be ruled out.

Based on the current study, undertriage seems present among PPU/PUB patients, possibly caused by the versatile symptomatology, the caller's description of the symptomatology, the telephone responders' perception of the symptomatology, and the structure of the Danish Index. No prehospital guidelines exist explaining how to handle these patients. In the hospital setting, however, early endoscopic therapy has been shown to reduce mortality and several time-dependent inhospital endoscopic national guidelines exist $[8,43]$. The initial assessment by the telephone responder is of great importance in securing efficient prehospital triage, as correct triage will reduce the time from onset of symptoms to initial on scene evaluation by health care professionals. At this point, rapid initial prehospital evaluation of the patient with objective measures of severity will further help clinical decision making and, if necessary, modulate the triage of the patient. Factors that can improve the prehospital care of patients with PPU/PUB include: improvements of the Danish Index, a structured training program for telephone responders in the EMCC, and the development of a fast track handling from the prehospital phase to the inhospital phase in order to improve this transition. Future studies should address these challenges.

\section{Conclusion}

Among patients calling 1-1-2, patients diagnosed with PPU/PUB had a lower proportion of highest level of triage and a higher proportion of lowest level of triage than FHQ patients. The 30-day mortality among patients diagnosed with PPU/PUB was comparable to the 30-day mortality among patients diagnosed with cardiac chest pain and trauma. This study suggests that undertriage is present among patients diagnosed with PPU/PUB. 


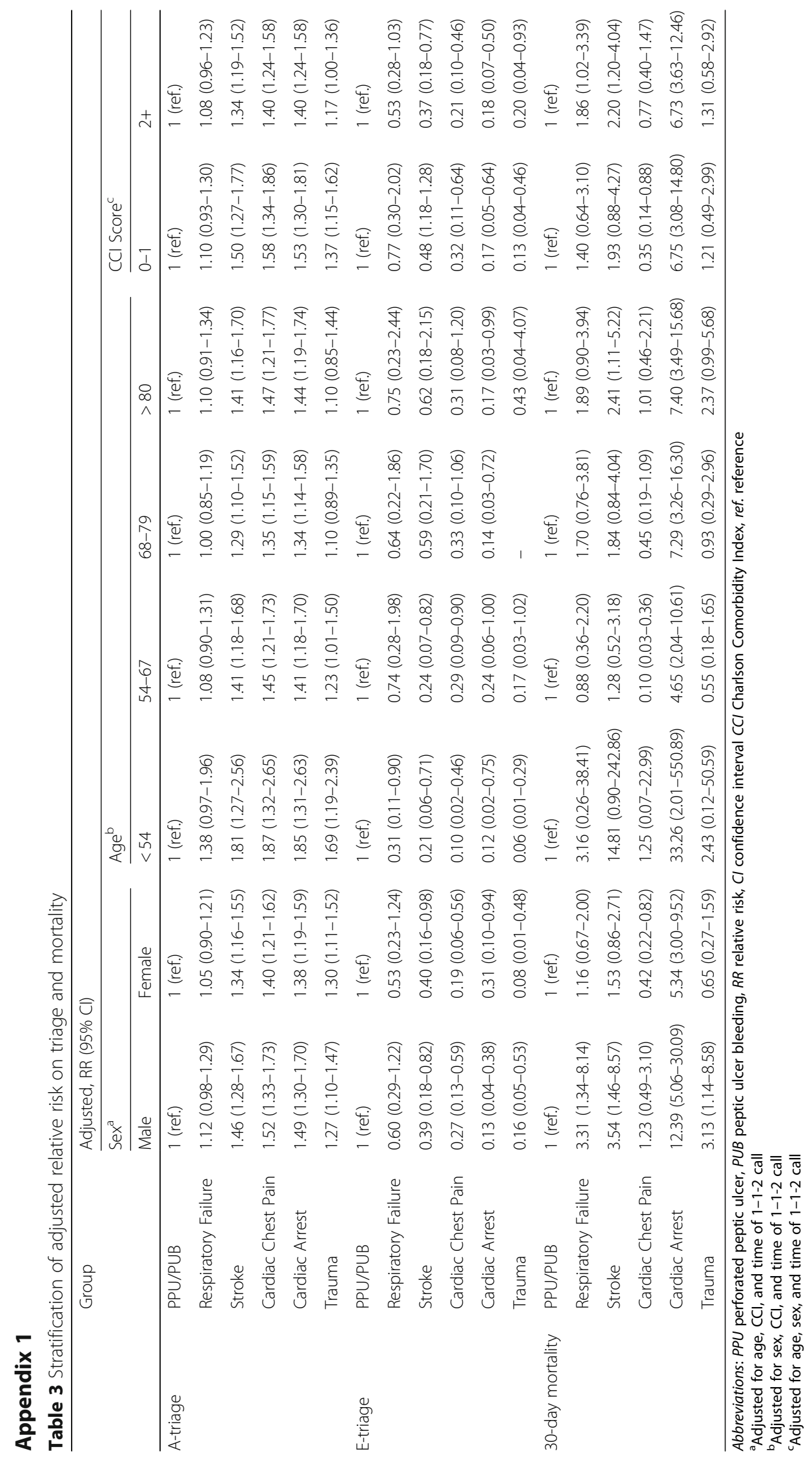


$\widehat{\mathrm{U}}$

ڤั.

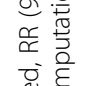

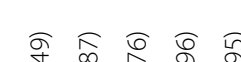

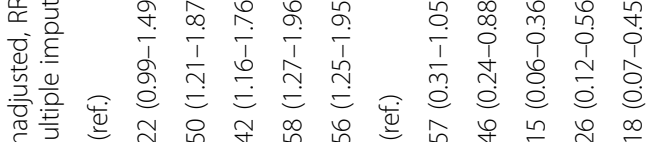

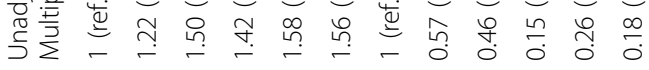

$\widehat{\cup}$

总

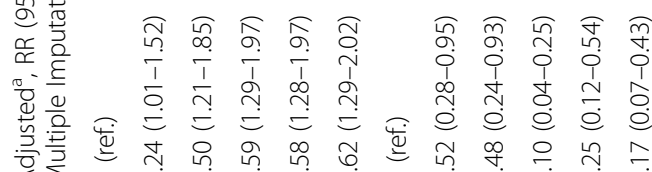

$\widehat{\cup}$

产

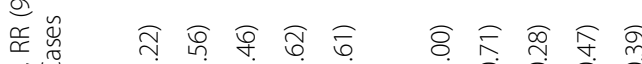

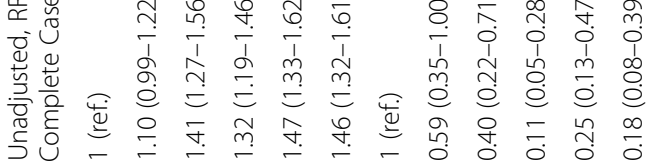

తิ

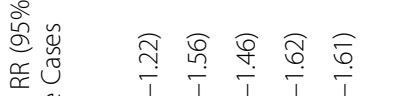

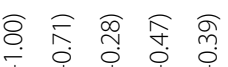

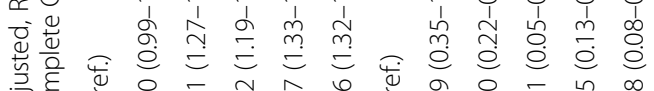

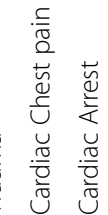

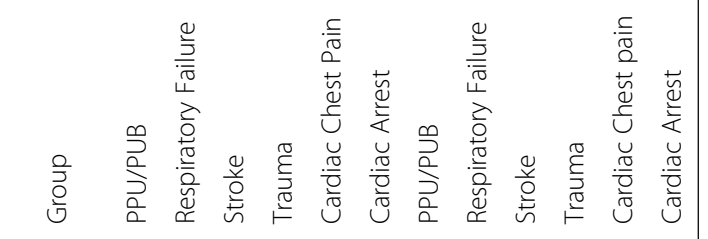

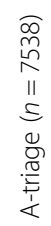

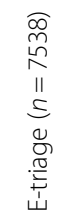

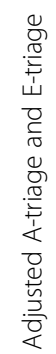

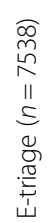




\section{Appendix 3}

Table 5 ICD-10 definitions

\begin{tabular}{|c|c|c|c|}
\hline & Diagnose & ICD-10 codes & $\begin{array}{l}\text { Frequency, } \\
\mathrm{n}(\%)\end{array}$ \\
\hline \multirow[t]{2}{*}{ PPU/PUB } & Perforated peptic ulcer & $\begin{array}{l}\text { DK251x, DK252x, DK255x, DK256x, DK261x, DK262x, DK265x, } \\
\text { DK266x, DK271x, DK272x, DK275x, DK276x }\end{array}$ & $216(2.49)$ \\
\hline & Peptic ulcer bleeding & DK250x, DK254x, DK260x, DK264x, DK270x, DK274x & $47(0.54)$ \\
\hline \multirow[t]{8}{*}{ Respiratory Failure } & Pulmonary embolism & $\mathrm{D} 126 \mathrm{x}$ & $208(2.40)$ \\
\hline & Heart failure & DI50.0, DI50.1, DI50.9 & $407(4.70)$ \\
\hline & Infection & DJ05x, DJ15x, DJ21x, DJ12.9, DJ40.9, DJ42x & $616(7.11)$ \\
\hline & Asthma & DJ45x & $228(2.63)$ \\
\hline & Pulmonary edema & DJ819 & $49(0.57)$ \\
\hline & Pneumothorax & DJ93x & $84(0.97)$ \\
\hline & Respiratory failure & DJ96.0, DJ96.1, DJ96.9, DR09.2 & $658(7.60)$ \\
\hline & Dyspnea & DR06.0 & $339(3.92)$ \\
\hline \multirow[t]{3}{*}{ Stroke } & Hemorrhage & DI60x, DI61x, DI62.1, D|62.9 & $363(4.19)$ \\
\hline & Cerebral infarction & D163x & $720(8.32)$ \\
\hline & $\begin{array}{l}\text { Transient cerebral ischemic } \\
\text { Attacks and related syndromes }\end{array}$ & DG45x & $605(6.99)$ \\
\hline \multirow[t]{3}{*}{ Cardiac Chest Pain } & Angina pectoris & DI20.0, DI20.1, DI20.8, DI20.9 & $670(7.74)$ \\
\hline & Acute myocardial infarction & $\mathrm{D} \mid 21 \mathrm{x}$ & $1121(12.95)$ \\
\hline & Other & DI23x, DI24.9 & $13(0.15)$ \\
\hline \multirow[t]{2}{*}{ Cardiac Arrest } & Cardiac arrest & DI46.0, DI46.1, DI46.9 & $1083(12.51)$ \\
\hline & Ventricular fibrillation & DI49.0 & $12(0.14)$ \\
\hline \multirow[t]{6}{*}{ Trauma } & Cranio-cerebral trauma & $\begin{array}{l}\text { DS021x, DS061, DS062x, DS063x, DS064x, DS065x, DS066x, } \\
\text { DS067x, DS068x, DS071x }\end{array}$ & $203(2.34)$ \\
\hline & Thorax injury & DS273x, DS274x, DS277x & $2(0.02)$ \\
\hline & Abdominal injury & DS352x, DS367x, DS368x, DS369x & $12(0.14)$ \\
\hline & Spine fracture & DS120x, DS127x & $22(0.25)$ \\
\hline & Poly-trauma & DS097x, DS383, DS757x, DT025x, DT029x, DT068x & 959 (11.08) \\
\hline & Burns & DT203x, DT213x, DT290x, DT293x, DT312x, DT317x, DT318x & $20(0.23)$ \\
\hline
\end{tabular}

Abbreviations: PPU perforated peptic ulcer, PUB peptic ulcer bleeding, ICD-10 International Classification of Diseases 10 th edition, $n$ number

\section{Abbreviations}

CCl: Charlson Comorbidity Index; Cl: Confidence Interval; CPR: Civil Personal Register; EMCC: Emergency Medical Communication Center; EMS: Emergency Medical Services; FHQ: First Hour Quintet; ICD-10: International Classification of Disease; IQR: Interquartile Ranges; N: Number; OR: Odds Ratio; PPU: Perforated Peptic Ulcer; PUB: Peptic Ulcer Bleeding; Ref:. Reference; RR: Relative Risk

\section{Acknowledgements}

I would like to acknowledge Ingunn S. Riddervold for the collection of the approval from the Danish Data Protection Agency, Vicki E. Vallund for her contribution regarding proofreading of the article, and the Biostatistical Advisory Service at Aarhus University for their contribution regarding imputation of missing data.

\section{Funding}

The research was funded by the Prehospital Emergency Medical Services, Central Denmark Region, Denmark.

\section{Authors' contributions}

$\mathrm{KB}$ participated in the design of the study, conceived the study, carried out the statistical calculations, and drafted the manuscript. KDF participated in the design of the study, the statistical calculations, and the draft of the manuscript. MTB helped to draft the manuscript. LN participated in the design of the study and the draft of the manuscript. All authors read and approved the final manuscript.

\section{Ethics approval and consent to participate}

The study was approved by the Danish Data Protection Agency (record number 1-16-02-207-15). See attached file "Ethics Approval".

\section{Consent for publication}

Approval by the local ethics committee and collection of informed consent are not required for observational studies.

\section{Competing interests}

The authors declare that they have no competing interests.

\section{Publisher's Note}

Springer Nature remains neutral with regard to jurisdictional claims in published maps and institutional affiliations.
The date sets used and analyzed during the current study are available from the corresponding author upon reasonable request. 


\section{Author details}

'Research Department, Prehospital Emergency Medical Services, Olof Palmes Allé 34, 8200 Aarhus N, Central Denmark Region, Denmark. ²Department of Anaesthesiology and Intensive Care, Aarhus University Hospital, Aarhus, Denmark.

\section{Received: 25 November 2017 Accepted: 27 March 2018}

Published online: 05 April 2018

\section{References}

1. Andersen MS, et al. Implementing a nationwide criteria-based emergency medical dispatch system: a register-based follow-up study. Scand J Trauma Resusc Emerg Med. 2013:21:53.

2. Andersen MS, et al. Preventable deaths following emergency medical dispatch-an audit study. Scand J Trauma Resusc Emerg Med. 2014:22:74

3. Kuisma M, et al. Prehospital mortality in an EMS system using medical priority dispatching: a community based cohort study. Resuscitation. 2004; 61(3):297-302.

4. Botker MT, et al. Long-term mortality of emergency medical services patients. Ann Emerg Med. 2017:70(3):366-73.

5. Moller TP, et al. Why and when citizens call for emergency help: an observational study of 211,193 medical emergency calls. Scand J Trauma Resusc Emerg Med. 2015;23:88

6. Lau JY, et al. Systematic review of the epidemiology of complicated peptic ulcer disease: incidence, recurrence, risk factors and mortality. Digestion. 2011:84(2):102-13.

7. Lassen A, Hallas J, Schaffalitzky de Muckadell OB. Complicated and uncomplicated peptic ulcers in a Danish county 1993-2002: a populationbased cohort study. Am J Gastroenterol. 2006;101(5):945-53.

8. Akut Kirurgi Databasen - National årsrapport 2015. 2015; Available from: https://www.sundhed.dk/content/cms/63/4663_\%C3\%A5rsrapport-2015akut-kiurgi-databasen-endelig-udgave2-22jan2016-3.pdf.

9. Cook DJ, et al. Endoscopic therapy for acute nonvariceal upper gastrointestinal hemorrhage: a meta-analysis. Gastroenterology. 1992;102(1): $139-48$

10. Gamst-Jensen H, Lippert FK, Egerod I. Under-triage in telephone consultation is related to non-normative symptom description and interpersonal communication: a mixed methods study. Scand J Trauma Resusc Emerg Med. 2017;25(1):52.

11. Bekendtgørelse om planlægning af sundhedsberedskabet og det præhospitale beredskab samt uddannelse af ambulancepersonale m.v. 2010, Retsinformation. https://www.retsinformation.dk/Forms/R0710.aspx?id= 180064.

12. Schmidt $M$, Pedersen $L$, Sorensen HT. The Danish civil registration system as a tool in epidemiology. Eur J Epidemiol. 2014;29(8):541-9.

13. Lynge E, Sandegaard JL, Rebolj M. The Danish National Patient Register. Scand J Public Health. 2011;39(7 Suppl):30-3.

14. Central Denmark Region - Health. [cited 2017 27. August]; Available from: http://www.rm.dk/om-os/english/

15. Henry JA, Reingold AL. Prehospital trauma systems reduce mortality in developing countries: a systematic review and meta-analysis. J Trauma Acute Care Surg. 2012;73(1):261-8.

16. Rosenstock SJ, et al. Improving quality of care in peptic ulcer bleeding: nationwide cohort study of 13,498 consecutive patients in the Danish clinical register of emergency surgery. Am J Gastroenterol. 2013;108(9):1449-57.

17. Krafft T, et al. European emergency data project (EED project): EMS databased health surveillance system. Eur J Pub Health. 2003;13(3 Suppl):85-90.

18. Cryer C, Langley JD. Developing valid indicators of injury incidence for "all injury". Inj Prev. 2006:12(3):202-7.

19. Gedeborg R, et al. Internationally comparable diagnosis-specific survival probabilities for calculation of the ICD-10-based injury severity score. J Trauma Acute Care Surg. 2014;76(2):358-65.

20. Thygesen SK, et al. The predictive value of ICD-10 diagnostic coding used to assess Charlson comorbidity index conditions in the population-based Danish National Registry of patients. BMC Med Res Methodol. 2011;11:83.

21. Sundboll J, et al. Positive predictive value of cardiovascular diagnoses in the Danish National Patient Registry: a validation study. BMJ Open. 2016;6(11):e012832.

22. Zou G. A modified poisson regression approach to prospective studies with binary data. Am J Epidemiol. 2004;159(7):702-6.

23. Klein JP, et al. Analyzing survival curves at a fixed point in time. Stat Med. 2007;26(24):4505-19.
24. White IR, Royston P, Wood AM. Multiple imputation using chained equations: issues and guidance for practice. Stat Med. 2011:30(4):377-99.

25. Efron B. Bootstrap methods: another look at the jackknife. Ann Statist. 1979; 7(1):1-26.

26. Palma $\mathrm{E}$, et al. Analysis of emergency medical services triage and dispatch errors by registered nurses in Italy. J Emerg Nurs. 2014;40(5):476-83.

27. Lu TC, et al. Preventable deaths in patients admitted from emergency department. Emerg Med J. 2006;23(6):452-5.

28. Nafsi $T$, et al. Audit of deaths less than a week after admission through an emergency department: how accurate was the ED diagnosis and were any deaths preventable? Emerg Med J. 2007:24(10):691-5.

29. Saltzherr TP, et al. Preventability of trauma deaths in a Dutch Level-1 trauma Centre. Injury. 2011:42(9):870-3.

30. Gijsenbergh F, Nieuwenhof A, Machiels K. Improving the first link in the chain of survival: the Antwerp experience. Eur J Emerg Med. 2003;10(3):189-94.

31. Calle PA, et al. Do victims of an out-of-hospital cardiac arrest benefit from a training program for emergency medical dispatchers? Resuscitation. 1997: 35(3):213-8.

32. Clawson JJ, et al. Effect of a comprehensive quality management process on compliance with protocol in an emergency medical dispatch center. Ann Emerg Med. 1998;32(5):578-84.

33. Terkelsen CJ, et al. Reduction of treatment delay in patients with STelevation myocardial infarction: impact of pre-hospital diagnosis and direct referral to primary percutanous coronary intervention. Eur Heart J. 2005; 26(8):770-7.

34. Terkelsen CJ, et al. Potential significance of spontaneous and interventional ST-changes in patients transferred for primary percutaneous coronary intervention: observations from the ST-MONitoring in acute myocardial infarction study (the MONAMI study). Eur Heart J. 2006;27(3):267-75.

35. Terkelsen CJ, et al. System delay and mortality among patients with STEMI treated with primary percutaneous coronary intervention. JAMA. 2010; 304(7):763-71

36. Terkelsen $\mathrm{CJ}$, et al. Health care system delay and heart failure in patients with ST-segment elevation myocardial infarction treated with primary percutaneous coronary intervention: follow-up of population-based medical registry data. Ann Intern Med. 2011;155(6):361-7.

37. Thang ND, et al. Characteristics of and outcome for patients with chest pain in relation to transport by the emergency medical services in a 20-year perspective. Am J Emerg Med. 2012;30(9):1788-95.

38. Thang ND, et al. Patients admitted to hospital with chest pain-changes in a 20-year perspective. Int J Cardiol. 2013;166(1):141-6.

39. Schmidt $M$, et al. The Danish National Patient Registry: a review of content, data quality, and research potential. Clin Epidemiol. 2015;7:449-90.

40. Botker MT, et al. Dyspnea, a high-risk symptom in patients suspected of myocardial infarction in the ambulance? A population-based follow-up study. Scand J Trauma Resusc Emerg Med. 2016;24:15.

41. Christensen EF, et al. Diagnosis and mortality in prehospital emergency patients transported to hospital: a population-based and registry-based cohort study. BMJ Open. 2016;6(7):e011558.

42. Moller TP, et al. The difficult medical emergency call: a register-based study of predictors and outcomes. Scand J Trauma Resusc Emerg Med. 2017;25(1):22.

43. Lin HJ, et al. Early or delayed endoscopy for patients with peptic ulcer bleeding. A prospective randomized study. J Clin Gastroenterol. 1996;22(4):267-71.

\section{Submit your next manuscript to BioMed Central and we will help you at every step:}

- We accept pre-submission inquiries

- Our selector tool helps you to find the most relevant journal

- We provide round the clock customer support

- Convenient online submission

- Thorough peer review

- Inclusion in PubMed and all major indexing services

- Maximum visibility for your research

Submit your manuscript at www.biomedcentral.com/submit 